\title{
Main Belt Comets as Clues to the Distribution of Water in the Early Solar System
}

\section{Lead Author:}

Karen J. Meech

University of Hawai'i, Institute for Astronomy meech@ifa.hawaii.edu

\section{Co-Authors/Endorsers:}

Julie Castillo-Rogez (JPL), Mathieu Choukroun (JPL), Gianrico Filacchione (INAF-IAPS), Olivier Hainaut (European Southern Observatory), Henry Hsieh (PSI), Ted Bergin (Univ. Michigan), Gary Huss (Univ. of Hawai i i), Jacqueline V. Keane Univ. of Hawai'i), Jan Kleyna (Univ. of Hawai ${ }^{i}$ ), Alice Lucchetti (INAF-Ast. Obs of Padova), Alessandro Morbidelli (Observatoire de la Côte d'Azur), Maurizio Pajola (INAF-Ast. Obs of Padova), Jian-Yang Li (PSI), Carol Raymond (JPL), Sean Raymond (Laboratoire d'Astrophysique de Bordeaux) 


\section{Abstract}

No one knows how water arrived at our planet, or if our solar system, with a planet possessing the necessary ingredients for life within the habitable zone, is a cosmic rarity. We do not know the role that the gas giants played in delivering essential materials to the habitable zone. The answers to these questions are contained in volatiles unaltered since the formation of the giant planets. To access this record, we need: (1) a population of icy bodies that faithfully records the history of volatile migration in the early solar system; (2) a source of volatiles that we can access affordably; (3) knowledge that the volatiles were not altered by aqueous interaction with their parent body; and (4) measurements from multiple chemical markers with sufficient precision to distinguish between original volatile reservoirs. Main belt comets (MBCs) are the perfect (and only) targets for addressing this science because they satisfy the criteria outlined above. MBCs are part of a large population of icy asteroids residing in the outer asteroid belt that have emerged as significant reservoirs of primordial water and potentially other volatiles. These icy asteroids may have formed in-situ or been dynamically implanted as the giant planets grew. Unlike short period or long period comets, they have remained on stable orbits within the asteroid belt since the era of planet formation or migration and preserve a record of their accretional environment.

\section{Introduction}

We have only one example of an inhabited world, namely Earth, with its thin veneer of water, the solvent essential to known life. Is a terrestrial planet like Earth that lies in the habitable zone and has the ingredients of habitability a common outcome of planet formation or an oddity that relied on a unique set of stochastic processes during the growth and subsequent evolution of our solar system? Ultimately, water originates in space, likely formed on dust grain surfaces via reactions with atoms inside cold molecular clouds (Tielens \& Hagen, 1982). Both water and organics are abundant ingredients in protoplanetary disks out of which solar systems form. In the disk, water is present both as a gas and a solid and is likely processed during planet formation. Water only exists as ice past the disk's snow line. The Earth formed in our solar system's inner protoplanetary disk where it's low mass would have made it hard to accrete much water in gaseous form.

Recent work on tungsten and molybdenum anomalies seen in non-carbonaceous and carbonaceous iron meteorites has suggested that these two reservoirs co-existed and were separate between 1-4 Myr after the formation for the first solar system solids (Kruijer et al., 2017). Kruijer et al. (2017) suggest that the mechanism for this separation was the early formation of Jupiter which opened a gap in the disk and prevented mixing by impeding the flow of icy material. These type of gaps are seen now in many protoplanetary disks as imaged by ALMA (Andrews, 2020).

Disk chemistry is responsible for the key chemical markers that can be used to trace the transport of water in the disk and this is transferred to the planetesimals as ice freezes on dust grains. The isotopic composition is imprinted on planetesimals when they form, but because of the dynamical re-arrangement during planet formation, the current location of small solar system bodies may not reflect their formation location. Orbital migration is a ubiquitous process in planet formation 1 . Some models predict significant migration that can scatter planetesimals inward from a

\footnotetext{
${ }^{1}$ It is not the intent of this white paper to review various scenarios for planet formation as it relates to the delivery of volatiles, but for a good review see Meech \& Raymond (2020).
} 
wide range of distances (Tsiganis et al., 2005; Walsh et al., 2011), whereas scattering may occur from a more limited region if the giant planets didn't migrate (Raymond \& Izidoro, 2017). The unique chemical and isotopic signatures that are imprinted on the icy material the is incorporated in the planetesimals can be used to understand where they formed.

Comets were long thought to be the most likely source of Earth's water (Owen \& Bar-Nun, 2001) because of the elevated D/H in Earth's oceans and the knowledge that D-enrichment occurs at low temperatures in the ISM (Herbst, 2003). However new models and new data, including Rosetta mission results from comet 67P, show that comets chemically similar to 67P could not have delivered the majority of volatiles because their $\mathrm{D} / \mathrm{H}$ isotopic signatures in water do not match that of Earth (Altwegg et al., 2015). Alternatively, there is another reservoir that we can sample that also probes early solar system history. Any material scattered into the inner solar system by the giant planets could have both delivered material to Earth and become trapped in the asteroid belt (where it exists today).

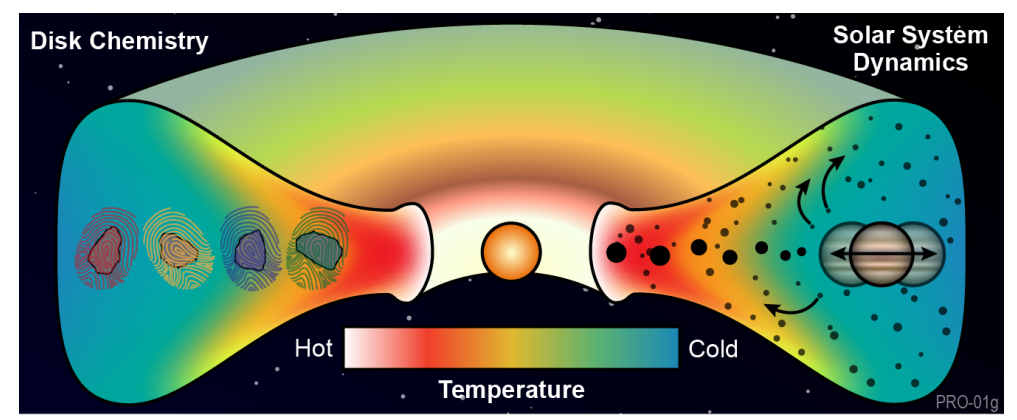

Figure 1: Protoplanetary disk chemical signatures are implanted on planetesimals as ices freeze near the midplane. This chemical signature is a sensitive measure of the planetesimal formation location. Subsequent planetesimal scattering as the giant planets grew will scramble this signature. The key to understanding where inner solar system volatiles originated is to measure the isotopes in a primitive reservoir of material that records the dynamical history during formation.

The only way to learn where Earth's water came from is to match the chemical fingerprints of inner solar system volatiles to a location in the protoplanetary disk. This approach can distinguish between competing models of solar system formation to pinpoint where terrestrial water came from and how it was delivered.

\section{Ice in the Asteroid Belt}

Direct evidence for water in the outer asteroid belt stems from the Herschel detection of water vapor from Ceres (Küppers et al., 2014) (coupled with water ice detections on Ceres' surface by Dawn; Combe et al., 2016) and a possible $\mathrm{H}_{2} \mathrm{O}$-frost signature on the asteroids 90 Antiope (Hargrove et al., 2015) and 24 Themis (Campins et al., 2010; Rivkin \& Emery, 2010). MBCs are the primitive representatives of accessible ice from the outer asteroid belt (Hsieh \& Jewitt, 2006; Jewitt et al. 2015). Orbiting in the outer asteroid belt since the early stages of solar system formation, they exhibit comet-like tails, attributed to outgassing of volatiles from ices in their interiors. The ices, preserved by a layer of dust, carry a signature of the early solar system. 
Active asteroids are small solar system bodies that exhibit comet-like mass loss yet have dynamically asteroidal orbits (Jewitt et al., 2015). Their orbits are largely dynamically stable over large (>100 Myr) timescales, unlike most comets (e.g., Haghighipour, 2009; Hsieh et al., 2012). Potential drivers of their observed activity include collisional dust ejection, rotational instability, and water-ice sublimation. Active asteroids whose activity is determined to be driven by sublimation are termed MBCs (see Snodgrass et al., 2017). On MBCs, activity may be triggered by impacts of meter-sized objects, which excavate sub-surface ice, thus initiating sublimation (Haghighipour et al., 2016). Most MBCs are related to collisional asteroid families (Hsieh et al., 2018b).

The Themis family represents the collisional remnants of an icy protoplanet a few $100 \mathrm{~km}$ in size. A weak or absent hydration signature suggests limited aqueous alteration in the Themis parent interior (Marsset et al., 2016) consistent with thermal models predicting a thick, unaltered outer layer of primitive ice and rock (Castillo-Rogez \& Schmidt, 2010). Stripping of Themis' outer layer formed a large fraction of the family members, including the MBCs, without reprocessing their volatile content (Rivkin et al., 2014). MBCs are samples from the unexplored icy asteroids small enough to have escaped hydrothermal processing, unlike Ceres, an icy asteroid that has suffered intensive aqueous alteration (Ammannito et al. 2016).

\section{Key MBC Investigations}

\subsection{Discovery}

As discussed above, only about a dozen MBCs are currently known, making it difficult to answer many key questions about the population, particularly about the extent and distribution of icy objects in the asteroid belt. Effectively using MBCs as compositional tracers of volatile material in the asteroid belt crucially depends on having a sufficiently large and representative sample population (Snodgrass et al., 2017). The small number of known MBCs also limits target and trajectory options for potential spacecraft missions to perform in-situ studies of MBCs (e.g., Meech \& Castillo-Rogez, 2015; Jones et al., 2018; Snodgrass et al., 2018).

Following the discovery of the first MBCs, there have been many efforts to find more MBCs (e.g., Waszczak et al., 2013; Chandler et al., 2018) using a variety of different approaches (see Appendix B.11; Hsieh et al., 2019), where the majority of currently known MBCs were discovered by the Pan-STARRS1 telescope (Hsieh et al., 2015). These searches for often weak MBC activity are typically limited by image sensitivity though, meaning that discovery rates of MBCs and other active asteroids should increase significantly during the upcoming Legacy Survey of Space and Time (LSST) to be conducted by the Vera C. Rubin Observatory and its $6.5 \mathrm{~m}$ effective diameter telescope, reaching approximately $r=24.5$ mag per visit, and the Nancy Grace Roman Space Telescope (see white papers on LSST by L. Jones et al. and the Roman Space Telescope by B. Holler et al.). That said, the development of effective active object detection algorithms and mechanisms for performing follow-up observations for confirmation and characterization of candidate MBCs will be essential for ensuring that discoveries are not missed and that MBCs are identified correctly.

\subsection{Physical Characterization}

Following discovery, the next key step to advancing our understanding of the MBC population is physical characterization of individual members of the population via observational studies. Such 
studies include but are not limited to regular monitoring of active apparitions to constrain activity onset and cessation times and confirm activity recurrence (a key distinguishing characteristic between MBCs with sublimation-driven activity and disrupted asteroids with non-sublimation-driven activity), dense time-series observations to obtain rotational periods and search for binarity, multifilter observations to constrain nucleus and coma composition, and inactive nucleus observations over a range of viewing geometries to constrain phase functions and absolute magnitudes (e.g., Agarwal et al., 2017; Hsieh et al., 2018a c). Such characterization observations require a range of observational facilities, from small-aperture telescopes (e.g., 2-m-class) able to monitor the activity of targets over long periods of time, to large-aperture telescopes (e.g., >8-m-class) able to collect high signal-to-noise data for precise physical characterization of faint inactive nuclei. We note that while wide-field survey data from LSST will be helpful, not all desired observational studies will be replicated by these data due to factors like LSST's short exposure times, sparse sampling, and noncontemporaneous multi-filter observations, meaning that targeted follow-up studies, even using relatively small aperture telescopes, will remain essential for MBC science even in the LSST era.

A key goal in MBC science is the direct spectroscopic detection of sublimation products that would confirm that the observed dust emission activity is driven sublimation of volatile ices. Unfortunately, MBCs are small (radii $<2 \mathrm{~km}$ ), and at the distance of the asteroid belt, have very low expected outgassing rates. Thus, it is not currently possible to detect gas from these objects with Earth-based telescopes (Snodgrass et al., 2017). Thirty-meter class facilities or potentially the James Webb Space Telescope (JWST) may be able to detect an outgassing signature from MBCs in the future; however, the precision isotope work that needs to be done to understand the story they can tell us about their origins will require in-situ measurements.

\subsection{Isotopic Measurements}

The most likely candidate for volatile delivery to the inner solar system is from external pollution from the outer solar system (Meech \& Raymond, 2020). However, this still leaves a wide range of possible distances from where the volatiles may have originated. The source location in the disk will depend upon where and when Jupiter's core formed. The key will be to match the signatures from volatiles in primitive objects that preserve the early solar system record and whose dynamical provenance is reasonably well understood. This region is the "wet" outer asteroid belt.

Isotopic measurements can be used as a fingerprint to trace the distance at which volatiles originated in the disk. However a single isotope is not a unique marker because gradients in disk chemistry are not uniform with either radial distance from the sun or height above the midplane (Willacy \& Woods, 2009) and there can be multiple locations in the disk with the same isotopic signature. However, different physical processes control the distribution of different isotopes, thus having multiple isotopes can constrain the formation conditions.

\subsubsection{Deuterium / Hydrogen and Oxygen Isotopes in Water}

In the outer protosolar nebula $\mathrm{D} / \mathrm{H}$ evolved from an initial supply of material that was enriched in D from low temperature ion-molecule reactions in the ISM (Herbst, 2003). Water enriched in D mixed with water that re-equilibrated via isotopic exchange with hydrogen from the warm inner nebula. Carbonaceous chondrite meteorites have a range of $\mathrm{D} / \mathrm{H}$ that best matches the $\mathrm{D} / \mathrm{H}$ of Earth's oceans and interior (Mottl et al., 2007; Hallis et al., 2015). 
Oxygen isotope variation in the disk has been attributed to either galactic chemical evolution or the more popular self-shielding mechanism (Lyons \& Young, 2005). With self-shielding, the three oxygen isotopes $\left({ }^{16} \mathrm{O},{ }^{17} \mathrm{O},{ }^{18} \mathrm{O}\right)$ have dramatically different abundances. The wavelengths necessary to dissociate the isotopologues of $\mathrm{CO}$ are distinct. At the edge of an accretion disk, UV light dissociates the same fraction of all the three isotopologues. But as the light penetrates into the cloud or disk, the photons that dissociate the more abundant ${ }^{12} \mathrm{C}^{16} \mathrm{O}$ are used up, so deeper in the cloud only ${ }^{12} \mathrm{C}^{17} \mathrm{O}$ and ${ }^{12} \mathrm{C}^{18} \mathrm{O}$ are dissociated. The resulting oxygen ions can either recombine into $\mathrm{CO}$ or combine with $\mathrm{H}_{2}$ to form $\mathrm{H}_{2} \mathrm{O}$. Deeper in the cloud, the $\mathrm{H}_{2} \mathrm{O}$ will be enriched in ${ }^{17} \mathrm{O}$ and ${ }^{18} \mathrm{O}$. If $\mathrm{MBCs}$ accreted in the outer disk and were subsequently trapped in the main belt, they should contain a higher abundance of heavy water.

\subsubsection{Nitrogen Isotopes}

Nitrogen fractionation in the disk is dominated by different physical mechanisms than for $\mathrm{D} / \mathrm{H}$ and oxygen, thus providing a third independent tracer. The primordial nebula and the Sun are significantly ${ }^{15} \mathrm{~N}$ depleted relative to Earth's atmosphere (Owen et al., 2001). Other reservoirs ( $\mathrm{CN}$ and $\mathrm{HCN}$ in comets, carbonaceous chondrite organics and Titan) are ${ }^{15} \mathrm{~N}$-enriched. This large fractionation may be inherited from the protosolar molecular cloud, where it is attributed to low-temperature ion-molecule reactions (Anders \& Grevesse, 1989) or it could have resulted from photochemical self-shielding effects in the protoplanetary disk (Lyons, 2012). The radial dependence on isotopic composition will differ significantly between these mechanisms.

\subsubsection{Noble Gases and Other Volatile Species}

Noble gases and other volatiles trapped in amorphous ice remain trapped in MBCs even after the ice crystallizes and can be used to interpret ice formation temperature. While only water can survive as pure ice in MBCs over the age of the solar system (Prialnik \& Rosenberg, 2009), other volatiles can be trapped in the water ice. These are initially trapped in amorphous ice, and the amount trapped is a sensitive function of trapping temperature and pressure (Bar-Nun et al., 1985, Yokochi et al., 2012), as is the isotopic fractionation.

It is not expected that today's MBCs retain any amorphous ice because their interior temperatures have warmed above the ice crystallization temperature. When amorphous ice crystallizes, a fraction of the trapped gases is retained in the water ice (possibly in the form of clathrates; Laufer et al., 2017) and is released only when the ice sublimates. This is seen consistently in laboratory experiments (Bar-Nun et al., 1988). Regardless of how gases remain trapped in the ice, it is clear that they do remain as seen observationally. For example, Comet Encke is expected to have a central temperature above the temperature for water ice crystallization (Herman \& Weissman, 1987). Nevertheless, it shows normal abundances of all comet species, including $\mathrm{NH}_{3}, \mathrm{HCN}$ and other compounds that sublimate at low temperatures (Roth et al., 2018), and is also $\mathrm{CO}_{2}$-rich (Reach et al., 2013), observational proof of volatile species survival.

\section{Landscape for Transformative Science in the Next Decade}

MBC science is closely related to efforts to trace the chemistry and physics of planet formation from the molecular cloud to a solar system. The next generation of sub-millimeter telescopes envisioned for the next decade will have $10 \times$ the sensitivity and resolution of the exquisite performance 
of ALMA (Murphy et al., 2018). These facilities will be able probe planet formation inside $10 \mathrm{au}$ (Andrews et al., 2018), unveiling the chemistry and structures in the habitable zones in nearby protoplanetary disks (McGuire et al., 2018; Ricci et al., 2018). Careful isotopic measurements of primitive volatiles in the outer asteroid belt gives us the ability to explore these past processes in our own solar system, although given the low expected sublimation rates at the heliocentric distances at which most MBCs are found, such detailed measurements will likely never be possible from Earth-based facilities and will instead need to be made in situ by a visiting spacecraft.

The combination of the expected discovery of many more MBCs by LSST and other wide-field surveys, and enhanced follow-up observational capabilities represented by the JWST and upcoming $30 \mathrm{~m}$-class telescopes in particular will present many exciting opportunities in the next decade to greatly advance our understanding about both the nature and history of volatile material in our own solar system as well as the conditions that led to the rise of life on Earth. Even more excit-

ingly, results from potential future investigations described in this white paper should also allow us to compare our solar system to other planetary systems, with implications for better understanding the likelihood of life arising in those other systems.

\section{References}

Agarwal, J., Jewitt, D., Mutchler, M., Weaver, H., \& Larson, S. 2017, Nature, 549, 357

Altwegg, K., Balsiger, H., Bar-Nun, A., et al. 2015, Science, 347, 1261952

Ammannito, E., DeSanctis, M. C., Ciarniello, M., et al. 2016, Science, 353, aaf4279

Anders, E., \& Grevesse, N. 1989, , 53, 197

Andrews, S. M. 2020, arXiv e-prints, arXiv:2001.05007

Andrews, S. M., et al. 2018, in Astronomical Society of the Pacific Conf. Series, Vol. 517, 137

Bar-Nun, A., Herman, G., Laufer, D., \& Rappaport, M. L. 1985, Icarus, 63, 317

Bar-Nun, A., Kleinfeld, I., \& Kochavi, E. 1988, , 38, 7749

Campins, H., Hargrove, K., Pinilla-Alonso, N., et al. 2010, Nature, 464, 1320

Castillo-Rogez, J. C., \& Schmidt, B. E. 2010, , 37, L10202

Chandler, C. O., Curtis, A. M., Mommert, M., et al. 2018, PASP, 130, 114502

Combe, J.-P., McCord, T. B., Tosi, F., et al. 2016, Science, 353, aaf3010

Haghighipour, N. 2009, Meteoritics and Planetary Science, 44, 1863

Haghighipour, N., Maindl, T. I., Schäfer, C., Speith, R., \& Dvorak, R. 2016, , 830, 22

Hallis, L. J., Huss, G. R., Nagashima, K., et al. 2015, Science, 350, 795

Hargrove, K. D., Emery, J. P., Campins, H., \& Kelley, M. S. P. 2015, Icarus, 254, 150

Herbst, E. 2003, Space Sci. Rev., 106, 293

Herman, G., \& Weissman, P. R. 1987, Icarus, 69, 314

Hsieh, H. H., Ishiguro, M., Knight, M. M., et al. 2018a, AJ, 156, 39

Hsieh, H. H., \& Jewitt, D. 2006, Science, 312, 561

Hsieh, H. H., Novaković, B., Kim, Y., \& Brasser, R. 2018b, AJ, 155, 96

Hsieh, H. H., Yang, B., Haghighipour, N., et al. 2012, AJ, 143, 104 
Hsieh, H. H., Denneau, L., Wainscoat, R. J., et al. 2015, Icarus, 248, 289

Hsieh, H. H., Ishiguro, M., Kim, Y., et al. 2018c, AJ, 156, 223

Hsieh, H. H., Bannister, M. T., Bolin, B. T., et al. 2019, arXiv e-prints, arXiv:1906.11346

Jewitt, D., Hsieh, H., \& Agarwal, J. 2015, Asteroids IV (Tucson, Univ. of Arizona Press), 221-241

Jones, G. H., Agarwal, J., Bowles, N., et al. 2018, Advances in Space Research, 62, 1921

Kruijer, T. S., et al. 2017, Proceedings of the National Academy of Science, 114, 6712

Küppers, M., O'Rourke, L., Bockelée-Morvan, D., et al. 2014, Nature, 505, 525

Laufer, D., Bar-Nun, A., \& Ninio Greenberg, A. 2017, , 469, S818

Lyons, J. R. 2012, in Lunar and Planetary Science Conference, 2858

Lyons, J. R., \& Young, E. D. 2005, Nature, 435, 317

Marsset, M., Vernazza, P., Birlan, M., et al. 2016, A\&A, 586, A15

McGuire, B. A., et al. 2018, in Astronomical Society of the Pacific Conf. Series, Vol. 517, 217

Meech, K. J., \& Castillo-Rogez, J. C. 2015, IAU General Assembly, 22, 2257859

Meech, K. J., \& Raymond, S. 2020, Origin of Earth's water: sources and constraints, 325-353

Mottl, M., Glazer, B., Kaiser, R., \& Meech, K. 2007, Chemie der Erde / Geochemistry, 67, 253

Murphy, E. J., et al. 2018, in Astronomical Society of the Pacific Conf. Series, Vol. 517, 3

Owen, T., Mahaffy, P. R., Niemann, H. B., Atreya, S., \& Wong, M. 2001, ApJ, 553, L77

Owen, T. C., \& Bar-Nun, A. 2001, Origins of Life and Evolution of the Biosphere, 31, 435

Prialnik, D., \& Rosenberg, E. D. 2009, , 399, L79

Raymond, S. N., \& Izidoro, A. 2017, Icarus, 297, 134

Reach, W. T., Kelley, M. S., \& Vaubaillon, J. 2013, Icarus, 226, 777

Ricci, L., et al. 2018, in Astronomical Society of the Pacific Conference Series, Vol. 517, 147

Rivkin, A. S., Asphaug, E., \& Bottke, W. F. 2014, Icarus, 243, 429

Rivkin, A. S., \& Emery, J. P. 2010, Nature, 464, 1322

Roth, N. X., Gibb, E. L., Bonev, B. P., et al. 2018, AJ, 156, 251

Snodgrass, C., Agarwal, J., Combi, M., et al. 2017, A\&A Rev., 25, 5

Snodgrass, C., Jones, G. H., Boehnhardt, H., et al. 2018, Advances in Space Research, 62, 1947

Tielens, A. G. G. M., \& Hagen, W. 1982, A\&A, 114, 245

Tsiganis, K., Gomes, R., Morbidelli, A., \& Levison, H. F. 2005, Nature, 435, 459

Walsh, K. J., Morbidelli, A., Raymond, S. N., et al. 2011, Nature, 475, 206

Waszczak, A., Ofek, E. O., Aharonson, O., et al. 2013, , 433, 3115

Willacy, K., \& Woods, P. M. 2009, , 703, 479

Yokochi, R., Marboeuf, U., Quirico, E., \& Schmitt, B. 2012, Icarus, 218, 760 